\title{
Evaluation and Outcomes of Women with a Breast Lump and a Normal Mammogram Result
}

\author{
Jennifer S. Haas, MD, MSPH, ${ }^{1,2}$ Celia P. Kaplan, DrPH, MA, ${ }^{3}$ Phyllis Brawarsky, MPH, ${ }^{1}$ \\ Karla Kerlikowske, $M D^{4}$ \\ 'Department of Medicine, Division of General Medicine and Primary Care, Brigham and Women's Hospital, Boston, Mass, USA; \\ ${ }^{2}$ Harvard Medical School, Harvard, Mass, USA; ${ }^{3}$ Department of Medicine, Medical Effectiveness Research Center, Division of General \\ Internal Medicine, University of California, San Francisco, Calif, USA; ${ }^{4}$ Department of Veterans Affairs, Departments of Epidemiology \\ and Biostatistics, and Medicine, General Internal Medicine Section, University of California, San Francisco, Calif, USA.
}

\begin{abstract}
BACKGROUND: Many women experience a breast lump. Clinical guidelines suggest that a normal mammogram result alone is not adequate to exclude a diagnosis of cancer.
\end{abstract}

OBJECTIVE: To examine the characteristics of women with a breast lump and a normal mammogram that were associated with receiving further evaluation, and to examine cancer outcomes.

DESIGN: Observational cohort.

PARTICIPANTS: Women aged 35 to 70 years who participated in a population-based mammography registry and who did not have a history of breast cancer noted at the time of their mammogram that they had a breast lump, and had a "normal" (Breast Imaging Reporting and Data System 1 or 2$)$ mammogram result $(n=771)$.

MEASUREMENTS: Telephone survey performed 6 months after the mammogram to ascertain information about evaluation. Cancer outcomes within 12 months of the index mammogram were confirmed through linkage with a cancer registry.

RESULTS: Only $56.9 \%$ of women reported receiving an adequate evaluation for their breast lump, including a subsequent clinical breast exam, a visit to a breast specialist, an ultrasound, a biopsy, or aspiration. Latinas were less likely than white women to have received adequate evaluation, as were obese women compared with normal-weight women, and uninsured women compared with women with insurance. Among women with at least 12 months of follow-up, 1.4\% were diagnosed with cancer.

CONCLUSIONS: Many women do not receive adequate evaluation for a recent breast lump. Interventions should be designed to improve the follow-up of women with this common clinical problem.

KEY WORDS: breast cancer; mammogram quality.

DOI: $10.1111 /$ j.1525-1497.2005.0149.x

J GEN INTERN MED 2005; 20:692-696.

$\mathrm{M}$ any women discover a breast lump that prompts them to seek medical attention. ${ }^{1}$ As approximately $10 \%$ of breast lumps ultimately lead to a diagnosis of breast cancer, ${ }^{1,2}$ it is important for women with a breast lump to receive appropriate evaluation. While mammography is commonly part of the evaluation of women with a breast lump, clinical guidelines and reviews suggest that a normal mammogram result alone is not an adequate evaluation, because $15 \%$ of women with palpable breast cancer have a normal mammogram result. ${ }^{3,4}$ These clinical recommendations are also supported by the observation that failure to diagnose breast cancer in a timely

The authors have no conflicts of interest to report.

Address correspondence and reprints request to Dr. Haas: Division of General Medicine and Primary Care, Brigham and Women's Hospital, 1620 Tremont Street, Boston, MA 02120-1613 (e-mail: jhaas@partners. org). manner is a common and costly cause of malpractice claims in the United States. ${ }^{5}$ The most common claim involves a premenopausal woman with a self-identified breast lump and a normal mammogram result and no further evaluation. ${ }^{6}$ Despite the existence of clinical guidelines, many women with a breast lump may not receive evaluation beyond mammography. ${ }^{7}$

The goal of this study was to examine the characteristics of women with a breast lump and a "normal" mammogram result (Breast Imaging Reporting and Data System (BIRADS) 1 or 2$)^{8}$ that were associated with receiving further evaluation and factors that were associated with the timeliness of evaluation. We hypothesized that women with a single breast lump or risk factors for breast cancer would be more likely to receive further evaluation, whereas women with a history of fibrocystic breast disease (or self-report of multiple lumps) and an average risk of developing breast cancer would be less likely to receive further evaluation. Through linkage with a cancer registry, we also examined the incidence of cancer in this population during the 12 months following the index mammogram.

\section{METHODS}

\section{Population}

The San Francisco Mammography Registry (SFMR; http:// mammography.ucsf.edu/SFMR/) is a population-based registry of women undergoing mammography. It is 1 of 7 registries participating in the National Cancer Institute Breast Cancer Surveillance Consortium. ${ }^{9}$ At each participating radiology facility, each woman completes a brief, scannable questionnaire at the time of her mammogram, which ascertains information about any breast symptoms experienced during the preceding 3 months, including a "new or unusual breast lump." Women were eligible for this study if they received a screening or diagnostic mammogram at any of 5 of the 11 SFMR mammography facilities between June 2001 and 2003, did not indicate at the time of their mammogram that they would be unwilling to be contacted in the future for research, were between 35 and 70 years of age, spoke English or Spanish, did not have a history of breast cancer or ductal carcinoma in situ (DCIS), noted at the time of their index mammogram that they had a breast lump within the preceding 3 months, and had a "normal" mammogram result (BIRADS 1 or 2). ${ }^{8}$ If a woman received more than 1 mammogram during the study period, she was eligible only for the first exam.

Received for publication March 3, 2005

and in revised form March 10, 2005

Accepted for publication March 10, 2005 
Potentially eligible women were sent an informational letter explaining the study and requesting their participation. This mailing included a prestamped "opt-out" postcard that a woman could return if she did not wish to be contacted. If no "opt-out" postcard was returned within 2 weeks of the mailing, attempts were made to contact the woman by telephone. When a woman was reached, verbal informed consent was obtained using a standard script. Women were enrolled between December 2001 and 2003. The research protocol was reviewed and approved by the institutional review boards of the participating institutions.

\section{Assessment}

Women who agreed to participate were asked to complete a single telephone survey approximately 6 months after their mammogram (mean 5.9 months). During this interview, women were asked about the characteristics of their breast lump (e.g., single or multiple lumps, location, month and year that the lump was first noted, and whether the lump was first detected by the patients, a health care provider, or someone else). Women were asked about sociodemographic characteristics, breast cancer risk factors, chronic medical conditions, subsequent evaluation, if any, and their understanding of the outcome of their assessment (i.e., received a normal or normal diagnosis, diagnosed with cancer or DCIS, still waiting for further testing).

\section{Variables}

Our principal outcome variable was a woman's report of her subsequent evaluation for her breast lump. Women were considered to have received an adequate evaluation if they reported a visit to a breast specialist, a subsequent clinical breast exam by any health care provider, or an ultrasound, fine-needle aspiration, or breast biopsy by the time of the telephone survey. Because some women may have received a fine-needle aspiration or breast biopsy prior to their mammogram, we also included these tests in our definition of "adequate" if they were reported to have occurred during the 2 months preceding the index mammogram. As a secondary outcome, we measured the time between the index mammogram and the first diagnostic procedure noted above among women who had received adequate evaluation. Finally, we examined whether a woman was subsequently diagnosed with cancer through linkage with the Northern California Surveillance Epidemiology and End Results (SEER) program. Cancer incidence was calculated for women who had at least 12 months between their index mammogram and cancer reporting for the facility where they received their mammogram to ensure $95 \%$ or greater completeness of cancer reporting.

Independent variables examined included age, race, and ethnicity (categorized as white, African American, Latina, and Asian or other), education (categorized as grades 0 to 12 or technical school; some college; college graduate, or postgraduate), country of birth (foreign- vs US-born), body mass index (categorized as normal if $<25 \mathrm{~kg} / \mathrm{m}^{2}$, overweight if 25 to $29.9 \mathrm{~kg} / \mathrm{m}^{2}$, obese if $\geq 30 \mathrm{~kg} / \mathrm{m}^{2}$ ), ${ }^{10}$ marital status (married or living with a partner vs other), insurance status (uninsured vs insured), the number of chronic medical conditions, a history of cancer (nonbreast), and breast health history. Breast health indicators included risk factors for breast cancer, in- cluding parity (nulliparous vs parous), prior breast biopsy (yes/no), first-degree family history of breast cancer (yes/no), and whether the woman experienced a single lump or multiple lumps or had a history of fibrocystic disease (yes/no).

\section{Data Analysis}

Multivariate logistic regression models were used to examine the associations between the significant independent variables and the receipt of an adequate evaluation. Cox models were used to examine the time to completion of an adequate evaluation. The dependent variable was time to the earliest test that indicated an "adequate" evaluation, measured in months. Women were censored on the date of their interview. These models were estimated with SAS (SAS Institute, Cary, NC) and SUDAAN (Research Triangle Institute, Research Triangle Park, NC). Independent variables were chosen on the basis of prior work and a priori hypotheses. ${ }^{1,3,4,7}$ All models also controlled for site of care.

\section{RESULTS}

\section{Response and Retention Rates}

Of the 1,703 women who were potentially eligible to participate in this study, 426 were found at the time of phone contact to be ineligible (could not speak English or Spanish ( $n=118$ ), denied any history of breast lump $(n=242)$, no telephone $(n=30)$, prior breast cancer $(n=3)$, other $(n=33)$. Of the 1,277 women who were eligible to participate, 771 women completed the survey (60.4\%), 246 (19.3\%) refused to participate, and 260 could not be contacted by telephone $(20.3 \%)$.

Women who completed the survey were younger than women who refused ( 48.9 vs 52.0 years, $P<.001$ ), were more likely to be white (61.7\% vs $37.7 \%$ ) or African American $(12.3 \%$ vs $9.3 \%$ ), and less likely to be Latina ( $16.2 \%$ vs $24.7 \%$ ) or Asian (9.7\% vs $28.4 \%$ to all $P<.001)$. Using data from the SFMR questionnaire completed by each woman at the time of her mammogram, we calculated the mean Gail score. Women who agreed to participate in this study had a mean Gail score of $2.9 \%$, women who refused $2.9 \%$, women who were ineligible (excluding the women who were already known to have breast cancer) $2.9 \%$, and those unreachable $2.8 \%(P=.81$ between groups). There were 2 breast cancers diagnosed in the women who refused to participate in the study or could not be contacted ( 2 of 506 or $0.40 \%$ of women).

\section{Description of the Sample}

The mean age of the sample was 49 years (Table 1). Fifty-five percent were pre-menopausal. The majority of women described their race and ethnicity as white, $16.2 \%$ described themselves as Latina, $12.3 \%$ as African American, and 9.7\% as Asian or other ethnicity. The majority of the sample had at least a college education. Eight percent of the sample was uninsured. Approximately half of the sample had been told that they had "fibrocystic breasts." Twenty-eight percent of women reported that they had more than one breast lump. Thirty-nine percent of the sample had a history of a breast biopsy, on average 50 months before the index mammogram. Seventeen percent of women reported a family history of a first-degree relative with breast cancer. This sample was at a relatively high risk of developing breast cancer: $81 \%$ had a 5 year risk of de- 
Table 1. Description of the Study Sample $(n=771)$

\begin{tabular}{|c|c|}
\hline & Number (\%) \\
\hline Mean age in years (range) & 48.9 (35 to 70$)$ \\
\hline \multicolumn{2}{|l|}{ Race and ethnicity* } \\
\hline White & $475(61.7)$ \\
\hline Latina & $125(16.2)$ \\
\hline African American & 95 (12.3) \\
\hline Asian/other & 75 (9.7) \\
\hline Foreign-born & $199(25.8)$ \\
\hline \multicolumn{2}{|l|}{ Education } \\
\hline Grade 0 to 12 or technical school & $143(18.5)$ \\
\hline Some college & $145(18.8)$ \\
\hline College graduate & $224(29.1)$ \\
\hline Postgraduate & $259(33.6)$ \\
\hline Married or living with partner & $432(56.0)$ \\
\hline Uninsured & $61(7.9)$ \\
\hline \multicolumn{2}{|l|}{ Breast health history } \\
\hline Diagnosed with fibrocystic breasts & $416(54.0)$ \\
\hline Currently has more than one lump & $218(28.3)$ \\
\hline $\begin{array}{l}\text { Family history of a 1st-degree relative } \\
\text { with breast cancer }\end{array}$ & $133(17.2)$ \\
\hline Prior breast biopsy & $302(39.2)$ \\
\hline Nulliparous & $304(39.4)$ \\
\hline \multicolumn{2}{|l|}{ Body mass index* } \\
\hline Normal & $468(60.8)$ \\
\hline Overweight & $175(22.7)$ \\
\hline Obese & $127(16.5)$ \\
\hline \multicolumn{2}{|l|}{ Chronic conditions } \\
\hline None & $448(58.1)$ \\
\hline Median number among women with at least 1 (range) & $1(1$ to 7$)$ \\
\hline Prior history of nonbreast cancer & $64(8.3)$ \\
\hline
\end{tabular}

*Data were missing for race $(\mathrm{n}=1)$, Body mass index $(\mathrm{n}=1)$.

veloping breast cancer of at least $1.67 \%$. The majority of women reported a normal body mass index and did not report any chronic conditions. Eight percent of women reported a history of nonbreast-related cancer (skin 24, cervix 10, thyroid 8, uterus 6 , colon 4 , other 12 ).

\section{Receipt of an Adequate Evaluation}

Only 56.9\% of women received adequate follow-up care (Table 2). Of these, the majority received a clinical breast exam. Among women who received adequate care, the median time to their evaluation was less than one month, and $36.0 \%$ of women had additional evaluation on the same day as their mammogram (or biopsy or fine needle aspiration (FNA) within 2 months before their mammogram). Fifteen women who had not yet received further evaluation reported that further evaluation was planned.

\section{Subsequent Diagnosis of Breast Cancer}

Of the 570 women with at least 12 months between their mammogram and complete cancer registry data, 8 (1.4\%) were diagnosed with breast cancer or DCIS. Of the 8 women with cancer or DCIS, 6 had received adequate care and 2 had not. Two of these 8 women had DCIS, 4 women were stage 1,1 woman was stage 2 , and 1 woman was stage 4 . The women with the stage 2 and stage 4 cancers both received adequate care. Among these women with a new diagnosis of breast cancer, the median time to diagnosis for women who received adequate evaluation was 4 days $(n=6)$, whereas for women who
Table 2. Care and Outcomes of Women in the Sample $(n=771)$

\begin{tabular}{lc}
\hline \hline & Number (\%) \\
\hline Adequacy of follow-up evaluation & \\
Adequate* & $439(56.9)$ \\
$\quad$ Received a clinical breast exam & $288(65.6)$ \\
Visit with a breast specialist & $93(21.2)$ \\
Received an ultrasound & $181(41.2)$ \\
Received a biopsy or FNA & $182(41.5)$ \\
Inadequate but patient reports plan for & $15(1.9)$ \\
further evaluation & \\
Inadequate & $317(41.2)$ \\
Median months to receipt of adequate & $0(0$ to 10$)$ \\
evaluation (range) & \\
Same day evaluation & $277(35.9)$ \\
Patient-reported outcome of evaluation & \\
Benign/normal & $713(93.1)$ \\
Cancer or carcinoma in situ & $8(1.0)$ \\
Still waiting for testing or further evaluation & $45(5.9)$ \\
\hline
\end{tabular}

*Adequate evaluation defined as a clinical breast exam subsequent to the index mammogram, a visit to a breast specialist, or receipt of an ultrasound, FNA, or biopsy (FNA or biopsy were also included in our criteria for adequate care if they occurred during the 2 months preceding the mammogram). Categories not mutually exclusive.

'Data missing for patient-reported outcome of evaluation ( $\mathrm{n}=5)$, months to receipt of adequate care $(\mathrm{n}=5)$.

FNA, fine needle aspiration.

had not received adequate evaluation the median time to diagnosis was 346 days ( $n=2 ; P=.13$ ).

\section{Reasons Why Women Did Not Receive an Adequate Evaluation}

Twenty-four women out of $332(7 \%)$ did not receive adequate care because they had not sought recommended care. Of these 24 women, the reasons for not seeking recommended care were: not believing that it was necessary $(n=10)$, not thinking that they had cancer $(n=15)$, being too busy $(n=11)$, and cost $(n=5)$.

\section{Factors Associated with Receiving an Adequate Evaluation}

Latinas were less likely than whites to receive adequate evaluation for their breast lump (odds ratio (OR) 0.69; 95\% confidence interval (95\% CI) 0.61 to 0.77 ) (Table 3). There were also differences in the adequacy of evaluation by level of education. Women who were obese were less likely to receive adequate evaluation for their breast lump. Women who were uninsured were also less likely to have received adequate evaluation than women with health insurance (OR 0.45; 95\% CI 0.21 to 0.99). Contrary to our hypothesis, women with fibrocystic breast disease were more likely to receive adequate evaluation than women who reported a single lump. While risk factors for breast cancer were not associated with adequate evaluation, women with a history of cancer at a nonbreast site were more likely to receive an adequate evaluation than women without a history of cancer (OR 2.27; 95\% CI 1.97 to 2.62). 
Table 3. Factors Associated with Receiving Adequate Evaluation $(n=770)$

\begin{tabular}{lc}
\hline \hline & $\begin{array}{c}\text { Adjusted Odds Ratio* } \\
\text { (95\% Confidence } \\
\text { Interval) }\end{array}$ \\
\hline $\begin{array}{l}\text { Race and ethnicity } \\
\text { White }\end{array}$ & 1 \\
Latina & $0.69(0.61$ to 0.77$)$ \\
African American & $0.86(0.63$ to 1.16$)$ \\
Asian/Other & $1.16(0.73$ to 1.85$)$ \\
Education & \\
O to 12 or technical school & $0.70(0.50$ to 0.99$)$ \\
Some college & $0.88(0.68$ to 1.14$)$ \\
College graduate & $0.75(0.57$ to 0.98$)$ \\
Post-graduate & 1 \\
Body mass index (BMI) & 1 \\
$\quad$ Normal & \\
Overweight & \\
Obese & $0.77(0.53$ to 1.10$)$ \\
Married or living with partner & $0.83(0.71$ to 0.98$)$ \\
$\quad$ (relative to others) & $1.23(0.92$ to 1.63$)$ \\
Uninsured (relative to insured) & \\
Breast cancer risk factors (relative & $0.45(0.21$ to 0.99$)$ \\
$\quad$ to those without the risk factor) & \\
$\quad$ History of fibrocystic disease & $1.38(1.26$ to 1.52$)$ \\
Multiple lumps & $1.31(0.60$ to 2.82$)$ \\
Family history of breast cancer & $1.01(0.65$ to 1.54$)$ \\
Prior breast biopsy & $1.03(0.80$ to 1.32$)$ \\
Nulliparous & $0.82(0.61$ to 1.12$)$ \\
Prior cancer (relative to those without & $2.27(1.97$ to 2.62$)$ \\
$\quad$ cancer) & \\
Number of chronic conditions & $1.13(0.96$ to 1.33$)$ \\
\hline
\end{tabular}

*Model adjusted for age, race/ethnicity, country of birth, level of education, BMI, marital status, insurance status, multiple lumps, history of fibrocystic breast disease, family history of breast cancer, prior breast biopsy, nulliparity, number of chronic conditions, prior history of cancer, and site of mammogram.

\section{Time to the Completion of an Adequate Evaluation}

As women aged, their time to the receipt of an adequate evaluation was longer (Table 4), with a mean of 0.5 months for women less than 50 , and 1.7 months for women older than 50 . The time to receipt of an adequate evaluation was approximately twice as long for women who were uninsured as for women who had health insurance.

\section{DISCUSSION}

This study suggests that many women with a breast lump or their health care providers are reassured by a "normal" mammogram result (BIRADS 1 or 2) and do not pursue further evaluation. Both women and their health care providers should be targeted for interventions to improve the quality of care for this common breast problem. The rate of adequate care for this clinical problem is similar to that for women who require short-term follow-up of an abnormal mammogram ${ }^{11}$ or have other breast complaints. ${ }^{7}$

Clinical guidelines and reviews that have focused on the evaluation of women with a breast lump have encouraged providers to pursue diagnostic evaluation beyond mammography regardless of clinical risk factors. ${ }^{3,4}$ About $15 \%$ of women with a palpable cancer have a mammogram that does not demonstrate an abnormal finding. ${ }^{12}$ The absolute risk of breast cancer in this cohort was $1.4 \%$, approximately 10 times higher than the rates of breast cancer among asymptomatic women
Table 4. Time to Completing an Adequate Evaluation $(n=764)^{*}$

\begin{tabular}{|c|c|}
\hline & $\begin{array}{c}\text { Adjusted Hazard Ratio } \\
\text { (95\% Confidence } \\
\text { Interval) }\end{array}$ \\
\hline Age & 0.85 (0.75 to 0.97$)$ \\
\hline \multicolumn{2}{|r|}{, } \\
\hline White & 1 \\
\hline Latina & $0.78(0.49$ to 1.23$)$ \\
\hline African American & $0.88(0.62$ to 1.24$)$ \\
\hline Asian/Other & 0.97 (0.63 to 1.48$)$ \\
\hline \multicolumn{2}{|l|}{ Body mass index (BMI) } \\
\hline Normal & 1 \\
\hline Overweight & $0.90(0.69$ to 1.18$)$ \\
\hline Obese & $0.86(0.64$ to 1.15$)$ \\
\hline $\begin{array}{l}\text { Married or living with partner } \\
\text { (relative to others) }\end{array}$ & $1.10(0.89$ to 1.36$)$ \\
\hline Uninsured (relative to insured) & $0.47(0.24$ to 0.93$)$ \\
\hline \multicolumn{2}{|l|}{$\begin{array}{l}\text { Breast health risk factors (relative } \\
\text { to those without) }\end{array}$} \\
\hline History of fibrocystic disease & $1.21(0.98$ to 1.49$)$ \\
\hline Multiple lumps & $1.16(0.94$ to 1.43$)$ \\
\hline Family history & $1.03(0.79$ to 1.34$)$ \\
\hline Prior biopsy & $1.04(0.84$ to 1.28$)$ \\
\hline Nulliparous & $0.87(0.70$ to 1.09$)$ \\
\hline Prior cancer (relative to those without cancer) & 1.35 (0.99 to 1.83$)$ \\
\hline Number of chronic conditions & $1.04(0.93$ to 1.16$)$ \\
\hline
\end{tabular}

with a normal mammogram result, depending on age. ${ }^{4} \mathrm{~A}$ breast lump is the most common breast symptom associated with cancer. ${ }^{1}$ Prior studies have found that up to $10 \%$ of breast lumps result in a diagnosis of breast cancer. ${ }^{1,2}$ Using this estimate for the premammography probability of breast cancer and an overall negative likelihood ratio for screening mammography of $0.15,{ }^{4}$ the posttest chance of breast cancer is $1.6 \%$, similar to our observed rate of $1.4 \%$. This study provides an estimate for the risk of cancer among women with a breast lump and a normal (BIRADS 1 or 2) mammogram result.

This work extends our prior work examining the subsequent care of women with an abnormal mammogram or clinical breast complaint by focusing in more detail on women with a breast lump. ${ }^{7}$ While our earlier work found that many women do not receive adequate care for their breast problems, the majority of those women had an abnormal mammogram result, so we were unable to specifically examine the evaluation of women with a breast lump. The present study is consistent with other work that examined reasons for diagnostic delay among patients newly diagnosed with breast cancer. ${ }^{13}$ Goodson and Moore ${ }^{13}$ found that $5 \%$ of women with cancer had presented with a breast lump and then received a normal mammogram result and had been "inappropriately" reassured by a physician, including both primary care providers and consultant surgeons. In this case series, inappropriate reassurance of women with this common clinical problem was the leading cause of delay in diagnosis.

Our finding that the uninsured are less likely to receive adequate follow-up for this common clinical problem is consistent with the literature that has demonstrated that the uninsured are more likely to present with late-stage cancer than are individuals with health coverage. ${ }^{14,15}$ Our finding that obese women were less likely to receive follow-up care is also 
supported by the literature. Prior work suggests that obese women use screening mammography at lower rates ${ }^{16,17}$ and have a greater risk of having a false-positive mammogram. ${ }^{18}$ Our study extends this information about the quality of care for obese women by suggesting that obese women are less likely to receive recommended care for a breast lump. Given the increasing prevalence of obesity in the United States, it is important to understand how obesity may affect clinical care. Prior work demonstrates that African-American women and Latinas are more likely to present with a higher stage of disease and have more adverse prognostic indicators than white women. ${ }^{19-22}$ Although our study did not demonstrate a difference in care between African Americans and whites, we did find that Latina women were less likely than whites to receive appropriate care.

Among women diagnosed with breast cancer following their presentation with a breast lump, those who had received further care by the time of our telephone survey demonstrated a trend $(P=.13)$ toward earlier diagnosis than women who did not receive further care. While women who received further care were typically diagnosed within a month, those who did not receive their diagnosis were approximately diagnosed 1 year after their mammogram. The median breast cancer volume doubling time is 157 days for women over the age of 50 years and 80 days for women younger than 50 years, suggesting that delays of this duration may be clinically meaningful. ${ }^{23}$ Because of the small sample of women diagnosed with breast cancer, these findings should be confirmed in a larger sample.

This study has several limitations. It was not designed to examine the care of women who did not receive a mammogram as part of the evaluation of their breast lump. Women with a dominant breast lump may receive a fine-needle aspiration biopsy or an ultrasound as the first diagnostic test for a breast lump. ${ }^{4}$ Because many of these women receive a subsequent mammogram, to exclude the possibility of a nonpalpable cancer, we categorized women as having received adequate care if they had received any further evaluation in the 2 months preceding their index mammogram. Although the cancer outcomes were validated using a cancer registry, data on subsequent evaluation are based on self-reports. In our prior work, we found that women can accurately recall this type of evaluation over a 6 months time period. ${ }^{24}$ Nonresponse bias might affect our results. We do not know whether women who did not respond to the survey had a similar risk for inadequate follow-up. Finally, $81 \%$ of the women included in this sample had a 5 year risk of developing breast cancer of at least $1.67 \%$. These results should be confirmed in a larger sample of lowrisk women.

This work suggests that the management of women with a breast lump could be improved. The publication and dissemination of clinical guidelines and reviews alone may not be enough to change physician or patient behavior. ${ }^{25}$ Working with local opinion leaders, providing performance feedback, or offering financial incentives are among the methods used to change physician management practices. ${ }^{26}$ Many women with a breast lump are at risk for receiving inadequate care, particularly women who may have limited access to the health care system. Inadequate care may result in a clinically important delay in the diagnosis of breast cancer. Future efforts should focus on improving the care recommended and received by women with this common medical problem.
This work was supported by an NCl-funded Breast Cancer Surveillance Consortium co-operative agreement (U01CA63740).

\section{REFERENCES}

1. Barton MB, Elmore JG, Fletcher SW. Breast symptoms among women enrolled in a health maintenance organization: frequency, evaluation, and outcome. Ann Intern Med. 1999;130:651-7.

2. Sterns EE. Age-related breast diagnosis. Can J Surg. 1992;35:41-5.

3. Cady B, Steele GD Jr., Morrow M, et al. Evaluation of common breast problems: guidance for primary care providers. CA Cancer J Clin. 1998; 48:49-63.

4. Kerlikowske K, Smith-Bindman R, Ljung BM, Grady D. Evaluation of abnormal mammography results and palpable breast abnormalities. Ann Intern Med. 2003;139:274-84.

5. Guthrie TH. Breast cancer litigation: an update with practice guidelines. Breast J. 1999;5:335-9.

6. Kern KA. Causes of breast cancer malpractice litigation. A 20-year civil court review. Arch Surg. 1992;127:542-6 (discussion 546-7).

7. Haas JS, Cook EF, Puopolo AL, Burstin HR, Brennan TA. Differences in the quality of care for women with an abnormal mammogram or breast complaint. J Gen Intern Med. 2000;15:321-8.

8. American College of Radiology. The American College of Radiology Breast Imaging Reporting and Data System (BI-RADS). 4th edn. Reston, Va: American College of Radiology; 2003.

9. Ballard-Barbash R, Taplin SH, Yankaskas BC, et al. Breast cancer surveillance consortium: a national mammography screening and outcomes database. Am J Roentgenol. 1997;169:1001-8.

10. Body Mass Index for Age, Vol. 2002. Centers of Disease Control.

11. Poon EG, Haas JS, Louise Puopolo A, et al. Communication factors in the follow-up of abnormal mammograms. J Gen Intern Med. 2004;19: 316-23.

12. Barlow WE, Lehman CD, Zheng $\mathbf{Y}$, et al. Performance of diagnostic mammography for women with signs or symptoms of breast cancer. J Natl Cancer Inst. 2002;94:1151-9.

13. Goodson WH III, Moore DH II. Causes of physician delay in the diagnosis of breast cancer. Arch Intern Med. 2002;162:1343-8.

14. Roetzheim RG, Pal N, Tennant C, et al. Effects of health insurance and race on early detection of cancer. J Natl Cancer Inst. 1999;91:1409-15.

15. Ayanian JZ, Kohler BA, Abe T, Epstein AM. The relation between health insurance coverage and clinical outcomes among women with breast cancer. N Engl J Med. 1993;329:326-31.

16. Wee CC, McCarthy EP, Davis RB, Phillips RS. Obesity and breast cancer screening. J Gen Intern Med. 2004;19:324-31.

17. Hunt KA, Sickles EA. Effect of obesity on screening mammography: outcomes analysis of 88,346 consecutive examinations. Am J Roentgenol. 2000;174:1251-5.

18. Elmore JG, Carney PA, Abraham LA, et al. The association between obesity and screening mammography accuracy. Arch Intern Med. 2004;164:1140-7.

19. Gwyn K, Bondy ML, Cohen DS, et al. Racial differences in diagnosis, treatment, and clinical delays in a population-based study of patients with newly diagnosed breast carcinoma. Cancer. 2004;100:1595-604.

20. Shavers VL, Harlan LC, Stevens JL. Racial/ethnic variation in clinical presentation, treatment, and survival among breast cancer patients under age 35. Cancer. 2003;97:134-47.

21. Zaloznik AJ. Breast cancer stage at diagnosis: caucasians versus hispanics. Breast Cancer Res Treat. 1997;42:121-4.

22. Bentley JR, Delfino RJ, Taylor TH, Howe S, Anton-Culver H. Differences in breast cancer stage at diagnosis between non-Hispanic white and Hispanic populations, San Diego County 1988-1993. Breast Cancer Res Treat. 1998;50:1-9.

23. Peer PG, van Dijck JA, Hendriks JH, Holland R, Verbeek AL. Agedependent growth rate of primary breast cancer. Cancer. 1993;71: 3547-51

24. Kaplan CP, Crane LA, Stewart S, Juarez-Reyes M. Factors affecting follow-up among low-income women with breast abnormalities. J Womens Health (Larchmt). 2004;13:195-206.

25. BRIDGE Study Group. Responses of primary health care professionals to UK national guidelines on the management and referral of women with breast conditions. J Eval Clin Pract. 2002;8:319-25.

26. Soumerai SB, McLaughlin TJ, Gurwitz JH, et al. Effect of local medical opinion leaders on quality of care for acute myocardial infarction: a randomized controlled trial. JAMA. 1998;279:1358-63. 